\title{
Effect of chronic ethanol consumption in female rats subjected to experimental sepsis
}

\author{
C.L. Castro ${ }^{1}$, A.S. Aguiar-Nemer ${ }^{2}$, H.C. Castro-Faria-Neto ${ }^{3}$, F.R. Barros ${ }^{1}$, \\ E.M.S. Rocha ${ }^{4}$ and V.A. Silva-Fonseca ${ }^{5}$ \\ ${ }^{1}$ Programa de Pós-Graduação em Patologia, Universidade Federal Fluminense, Niterói, RJ, Brasil \\ ${ }^{2}$ Departamento de Nutrição, Instituto de Ciências Biológicas, Universidade Federal de Juiz de Fora, Juiz de Fora, MG, Brasil \\ ${ }^{3}$ Laboratório de Imunofarmacologia, Instituto Oswaldo Cruz, FIOCRUZ, Rio de Janeiro, RJ, Brasil \\ ${ }^{4}$ Departamento de Microbiologia e Parasitologia, Universidade Federal Fluminense, Niterói, RJ, Brasil \\ ${ }^{5}$ Departamento de Fisiologia e Farmacologia, Universidade Federal Fluminense, Niterói, RJ, Brasil
}

\begin{abstract}
The objective of this research was to evaluate the interference of ethanol consumption by female rats with cytokines involved in the sepsis process and its correlation with mortality, the main outcome of sepsis. Female Wistar rats in estrus phase were evaluated in three experiments. Experiment $1(n=40)$ was performed to determine survival rates. Experiment $2(n=69)$ was designed for biochemical analysis, measurement of cytokine and estrogen levels before and after sepsis, and experiment 3 $(n=10)$ was performed to evaluate bacterial growth by colony counts of peritoneal fluid. In all experiments, treated animals were exposed to a $10 \%$ ethanol/water solution $(\mathrm{v} / \mathrm{v})$ as the single drinking source, while untreated animals were given tap water. After 4 weeks, sepsis was induced in the rats by ip injection of feces. In experiment 1, mortality in ethanol-exposed animals was delayed compared with those that drank water $(48 \mathrm{~h} ; \mathrm{P}=0.0001)$. Experiment 2 showed increased tumor necrosis factor alpha (TNF- $\alpha$ ) and decreased interleukin-6 (IL-6) and macrophage migration inhibitory factor in septic animals exposed to ethanol compared to septic animals not exposed. Sepsis also increased TNF- $\alpha$ and IL- 6 levels in both ethanol- and waterexposed groups. Biochemical analysis showed higher creatinine, alanine aminotransferase and aspartate aminotransferase and decreased glucose levels in septic animals that were exposed to ethanol. In experiment 3 , septic animals exposed to ethanol showed decreased numbers of colony-forming units than septic animals exposed to water. These results suggest that ethanol consumption delays the mortality of female rats in estrus phase after sepsis induction. Female characteristics, most probably sex hormones, may be involved in cytokine expression.
\end{abstract}

Key words: Alcohol; Sepsis; Cytokines; Estrogens

\section{Introduction}

Ethanol is known to have a variety of effects on immune function, including decreased lymphocyte responsiveness to mitogen, decreased neutrophil chemotactic and phagocytic functions, and alteration of cytokine production by lymphocytes and macrophages (1). Extensive evidence indicates that ethanol consumption affects human health. These effects are complex and depend on many factors, such as patterns of drinking (chronic or acute), the amount of consumed ethanol (moderate or excessive), the body organ or system and the gender of the user. Chronic alcohol abuse, defined as $120-150 \mathrm{~g}$ or $\geqslant 8$ drinks per day, has been associated with immunosuppression and increased morbidity and mortality. The lowest death rate correlates with low to moderate amounts of ethanol, at 15$45 \mathrm{~g}$ or 1-3 drinks per day, while abstaining from ethanol or excessive drinking are both associated with higher mortality (2).

An increased predisposition to infection among patients with problems of alcohol use may mediate an association with sepsis (3). Despite recent improvements in intensive care and antibiotics, sepsis remains associated with a high mortality rate, possibly because of its diverse etiology and comorbidities. Sepsis is defined according to the clinical signs of a systemic response to infection. The term "severe sepsis" refers to the sepsisassociated failure of multiple organ systems (4). Between

Correspondence: V.A. Silva-Fonseca, Rua Hernani Melo, 101, 24210-130 Niterói, RJ, Brasil. Fax: +55-21-2629-2401. E-mail: vilma91@yahoo.com.br 
20 and $50 \%$ of patients with sepsis die and, in those who survive, sepsis is associated with reduced quality of life (5). Extensive efforts have been devoted to the prediction of mortality during sepsis. The initial hyperinflammatory response to bacteria can lead to septic shock. Risk factors for septic shock include advanced age, diminished physical performance, presence of pathogens, low platelet count, leukocytosis or leukopenia, low albumin concentration, increased levels of liver enzymes, and high plasma creatinine concentrations, which are very common in alcoholics. Septic shock is characterized by an over-production of cytokines, as shown in numerous clinical trials conducted with agents that block the inflammatory cascade. However, death from sepsis is not always attributable to an overstimulated immune system. Several observations demonstrated that as sepsis persists, there is a shift toward an anti-inflammatory immunosuppressive state (6).

It is increasingly recognized that gender may be a major factor in the outcome of patients after stress conditions such as trauma and sepsis. Findings from both clinical studies and experimental animal models suggest that women are more tolerant to major injuries than men (7-10) and sex steroids seem to be responsible for this gender-specific outcome (11).

However, medical literature in female subjects is scarce, probably because of hormonal alterations and consequent variability. As for the effects of ethanol, a few studies suggest that it would significantly alter sex hormone levels and function in exposed subjects (12).

The hypothesis of the present study was that ethanol interferes with the course of sepsis in female rats. Cytokines involved in the sepsis process and the interference of ethanol consumption on their level were studied, and their correlation with mortality, the main outcome of sepsis, was evaluated in female rats.

\section{Material and Methods}

\section{Animals}

The study was approved by the Animal Research Ethics Committe of Universidade Federal Fluminense (\#00115-09). Female Wistar (Rattus novergicus), 4 to 6 weeks old (150-170 g), were used. The animals were maintained on 12-h light-dark cycles with free access to food and liquid (water or ethanol). Animals exposed to ethanol received $10 \%$ ethanol/water solution $(\mathrm{v} / \mathrm{v} ; 10 \mathrm{~mL}$ ethanol $+90 \mathrm{~mL}$ water) daily for 4 weeks. Liquid and food consumption as well as body weight were measured every $48 \mathrm{~h}$.

\section{Septic procedure}

After 4 weeks of ethanol consumption, samples of vaginal cells were taken before initiation of the sepsis procedure and cardiac puncture. All female rats had pipettes with saline solution inserted $4 \mathrm{~mm}$ into the vagina. The pipettes were then pressed on microscope slides, and the cells were stained with methylene blue and examined under light microscopy. Estrus phases were determined by relative cell densities as follows. Diestrus was identified by predominance of small cells (leukocytes) as well as larger, round, nucleated epithelial cells and cornified epithelial cells; diestrus II by leukocytes and round, nucleated epithelial cells with few cornified epithelial cells; proestrus by round, nucleated epithelial cells; and estrus by cornified epithelial cells. Sepsis was induced in animals in the estrus phase by injecting fresh feces in the peritoneum.

To prepare the feces solution, random animals were sacrificed for removal of feces from the large intestine. The material was collected in conical polypropylene centrifuge tubes with a capacity of $50 \mathrm{~mL}$. Then, saline was added to feces dilution $(15 \mathrm{~mL}$ saline to each $5000 \mathrm{~mL}$ feces) and the final solution was centrifuged at $215 \mathrm{~g}$ for $5 \mathrm{~min}$. The supernatant was removed and injected intraperitoneally (ip) at $1 \mathrm{~mL} / 100 \mathrm{~g}$ body weight (13). Animals that drank water underwent the same technique described above after 4 weeks.

\section{Experiment 1: subchronic ethanol consumption and survival analysis}

In experiment 1, the animals (150-169 g) were randomly divided into two groups. A sepsis group consisting of 20 animals receiving water, and an ethanol sepsis group consisting of 20 animals receiving a 10\% ethanol/water solution. Four weeks later, polymicrobial sepsis was induced in both groups of animals in estrus phase. Eight hours after sepsis induction, when the animals showed clinical signals of sepsis (ruffled fur, hypothermia, lacrimation, lethargy), a combination of the antibiotics imipenem and cilastatin $(8 \mathrm{mg} / \mathrm{kg}$ ) was injected subcutaneously to increase the survival rate. Animals were observed after septic induction every $8 \mathrm{~h}$ for detection of mortality.

\section{Experiment 2: subchronic ethanol consumption and immune response in sepsis}

In experiment 2, rats (4 to 6 weeks old and weighing $150-160 \mathrm{~g}$ ) were used to evaluate the effects of ethanol consumption on biochemical parameters, cytokine and estrogen levels after sepsis induction. The animals were randomly assigned to one of four groups. Controls received water and were challenged with ip saline $(n=12)$. The septic group received water and was challenged with ip feces solution $(n=20)$. Two of the groups received $10 \%$ ethanol/water solution. The ethanol group was challenged with saline $(n=12)$, and the ethanol septic group was challenged with ip feces solution $(n=25)$. Four weeks later, the septic and ethanol septic groups underwent sepsis induction while the control and ethanol groups received normal saline ip. Whole blood 
was obtained by cardiac puncture $8 \mathrm{~h}$ after sepsis induction or saline injection in animals in estrus phase.

\section{Serum collection and storage}

For the cardiac puncture procedure, rats were anesthetized with $112.5 \mathrm{mg} / \mathrm{kg}$ ketamine and $7.5 \mathrm{mg} / \mathrm{kg}$ xylazine ip. Serum was separated by centrifugation at $1700 \mathrm{~g}$ and samples were immediately frozen and stored $\left(-80^{\circ} \mathrm{C}\right)$ until assayed.

\section{Biochemical analysis}

Biochemical tests were performed on a semiautomatic spectrophotometer brand model BYO2000 (Bioplus, Brazil). The following biochemical parameters were analyzed: alanine aminotransferase (ALT), aspartate aminotransferase (AST), albumin, creatinine, glucose, total protein, and urea. Serum ALT and AST were assayed by a kinetic method and serum creatinine, albumin, glucose, total protein, and urea by a colorimetric method. All tests used diagnostic reagent kits from Labtest Ltd., Brazil.

\section{Cytokines and estrogen analysis}

Pro-inflammatory [TNF- $\alpha, \quad$ IL- 6 and macrophage migration inhibitory factor (MIF)] and anti-inflammatory (TGF- $\beta$, IL-10 and IL-13) cytokines and female sex steroid hormone $17 \beta$-estradiol (E2) levels present in blood serum were quantified by ELISA (DuoSet Kit/R\&D Systems, USA) according to manufacturer's specifications. Data were analyzed using the SoftMax Pro 5 program (USA).

\section{Experiment 3: estimation of the number of bacterial colonies in peritoneal fluid}

Peritoneal fluid was obtained from 10 randomly chosen rats by washing the peritoneal cavity with $4 \mathrm{~mL}$ $0.9 \%$ saline. One animal was from the control group and 1 from the ethanol group; 4 animals were from the septic group and 4 from the ethanol septic group. Animals had ad libitum access to liquids and food for 4 weeks prior to induction of sepsis in the septic and ethanol septic groups as described above. For the analysis of bacterial growth, trypticase soy agar Petri plates were used. Serial 10-fold dilutions were prepared at $10^{-2}$ and $10^{-4}$. Plates were incubated at $37^{\circ} \mathrm{C}$ for $24 \mathrm{~h}$ and bacterial growth was evaluated from colony counts using the formula colonyforming units $(\mathrm{CFU})=$ colonies $\times$ dilution $/ 10 \mu \mathrm{L}$.

\section{Statistical analysis}

Data were analyzed with the GraphPad Prism 4.0 (USA) statistical program and are reported as means $\pm S E$. One-way ANOVA followed by the Student $t$-test were used to compare body weight data. Log rank was used to compare survival proportions and KruskalWallis and Mann-Whitney tests for comparisons of the cytokines, estrogen and biochemistry groups. A P value $\leq 0.05$ was considered to be significant.

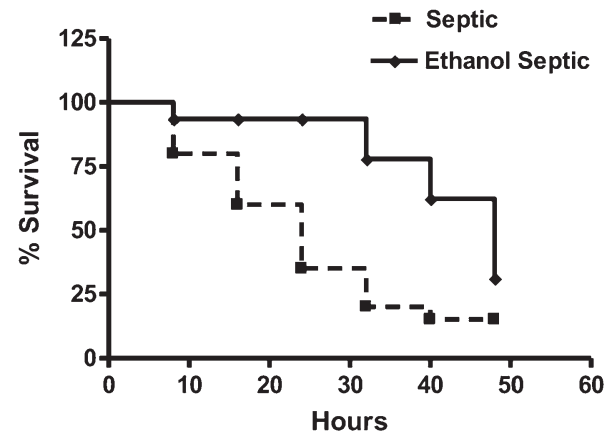

Figure 1. Ethanol consumption protects female rats against sepsis. The septic group $(n=20)$ received water and the ethanol septic group $(n=20)$ received $10 \%$ ethanol solution for 4 weeks. Animals in estrus phase were challenged with feces injected intraperitoneally at $48 \mathrm{~h} . \mathrm{P}=0.0001$, log rank test.

\section{Results}

\section{Experiment 1: ethanol consumption and survival analysis}

Daily ethanol consumption averaged $10.9 \pm 1.9 \mathrm{~g} / \mathrm{kg}$ in the exposed groups. We did not observe differences in body weight between the groups (control: $203.3 \pm 22.4 \mathrm{~g}$ ethanol: $181.2 \pm 23.9$; septic: $201.4 \pm 23.8 \mathrm{~g}$; ethanol septic: $184.4 \pm 23.7 \mathrm{~g}, \mathrm{P}>0.05)$. Twenty-four hours after sepsis induction, 13 animals in the septic group (65\%) and 2 animals in the ethanol septic group (13\%) had already died. After $48 \mathrm{~h}$, the mortality rate was 17 animals in the septic group (85\%) and 14 animals in the ethanol septic group $(70 \%$; Figure 1). The difference in the mortality rates in these groups reached statistical significance at $48 \mathrm{~h}(\mathrm{P}=0.0001$, log rank test $)$.

\section{Experiment 2: immune response in sepsis}

Cytokines. Analysis of pro- and anti-inflammatory serum cytokines $8 \mathrm{~h}$ after sepsis induction demonstrated that sepsis increased the levels of TNF- $\alpha$ and IL-6. There was also a significant increase in the levels of the proinflammatory cytokine TNF- $\alpha$ in the ethanol septic group compared with the septic group $(P=0.02)$ and a decrease in the levels of the proinflammatory cytokines IL-6 and MIF in the ethanol septic group compared with the septic group, demonstrating that ethanol consumption altered the pattern of cytokine production after sepsis. There was no difference in IL-10 levels in the septic and the ethanol septic groups. No differences were observed in the levels of the anti-inflammatory cytokines TGF- $\beta$ and IL-13 in the groups (Figure 2).

\section{Biochemical analysis}

Liver function was evaluated by ALT and AST levels. The ethanol septic group showed higher levels of these enzymes. The differences in ALT levels in the ethanol septic and the ethanol groups and AST levels in the 

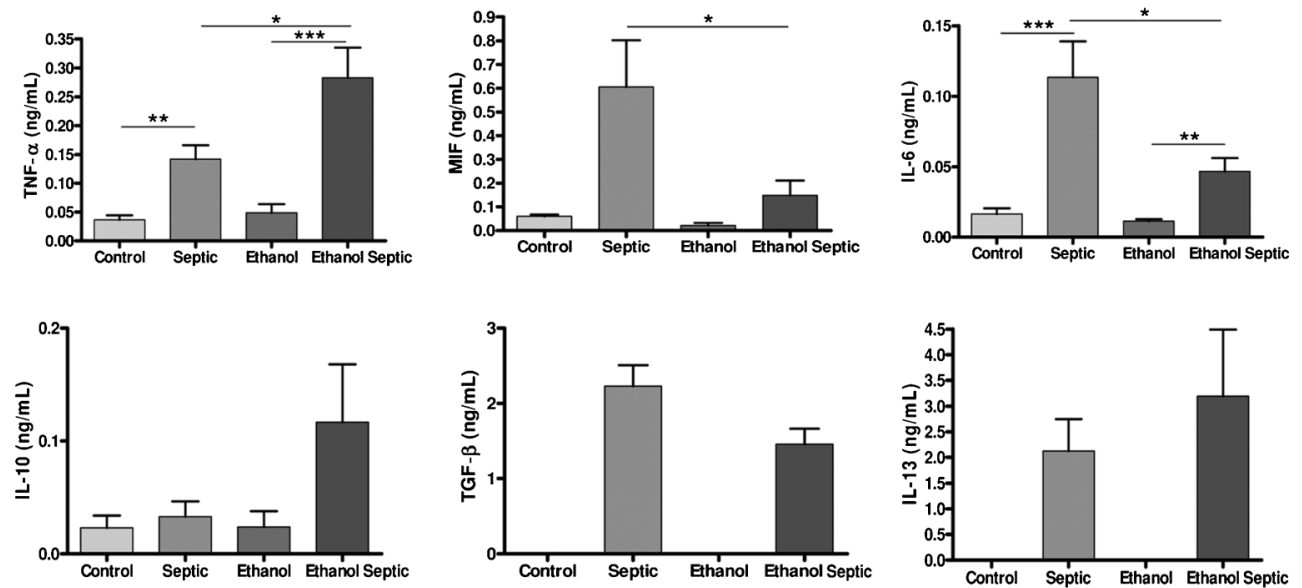

Figure 2. Ethanol consumption alters pro-inflammatory cytokine response after sepsis. Ethanol consumption did not interfere with antiinflammatory cytokine response after sepsis. Control $(n=12)$ and septic $(n=20)$ groups received water, and ethanol $(n=12)$ and ethanol septic $(n=25)$ groups received $10 \%$ ethanol solution for 4 weeks. Septic and ethanol septic groups underwent septic procedure while control and ethanol groups received normal saline injected intraperitoneally. Whole blood was obtained by cardiac puncture $8 \mathrm{~h}$ after sepsis induction or saline injection in animals in estrus phase. Data are reported as means $\pm \mathrm{SE}$ from at least 12 animals. Tumor necrosis alpha $(\mathrm{TNF}-\alpha)$ : ${ }^{*} \mathrm{P}=0.02 ;{ }^{* *} \mathrm{P}=0.004 ;{ }^{* * *} \mathrm{P}<0.0001$. Macrophage migration inhibitor factor (MIF): ${ }^{*} \mathrm{P}=0.03$. Interleukin 6 (IL6): ${ }^{*} \mathrm{P}=0.04 ;{ }^{* \star} \mathrm{P}=0.003 ;{ }^{* \star *} \mathrm{P}=0.0005$ (Mann-Whitney test). TNF- $\alpha$ : $\mathrm{P}<0.0001 ;$ IL-6: $\mathrm{P}<0.0001 ;$ MIF: $\mathrm{P}=0.041$ (Kruskal-Wallis). No significant differences were found by Kruskal-Wallis (IL-10) and Mann-Whitney [transforming growth factor beta (TGF- $\beta$ ); IL-13] tests.

ethanol septic and the septic groups were statistically significant (Figure 3). Creatinine analysis showed an increase in the ethanol septic group compared with the ethanol and the septic groups. There was also a significant difference between hypoglycemia in animals with sepsis that consumed ethanol compared to the ethanol and the septic groups. No differences in proteins, urea and albumin levels were noted between the groups (Figure 4).

\section{Experiment 3: estimation of the number of bacterial colonies in peritoneal fluid}

As expected, there was no bacterial growth in the control and the ethanol groups. In all animals subjected to sepsis, there was a positive detection of growth. The bacterial colonies in both groups were too numerous to count at a dilution of $10^{-2}$. At a dilution of $10^{-4}$, the median numbers of colonies were 35,000 CFU for control septic animals and $5000 \mathrm{CFU}$ for ethanol septic animals.

\section{Discussion}

This study suggests that ethanol consumption delays the mortality of female rats after sepsis induction. The survival advantage was associated with increases in the pro-inflammatory cytokine TNF- $\alpha$ and decreases in IL-6 and MIF levels. However, it was not associated with differences in the anti-inflammatory cytokines IL-10, TGF$\beta$ and IL-13.

We have reported on the same sepsis induction model in male rats exposed to $10 \%$ ethanol/water as the only
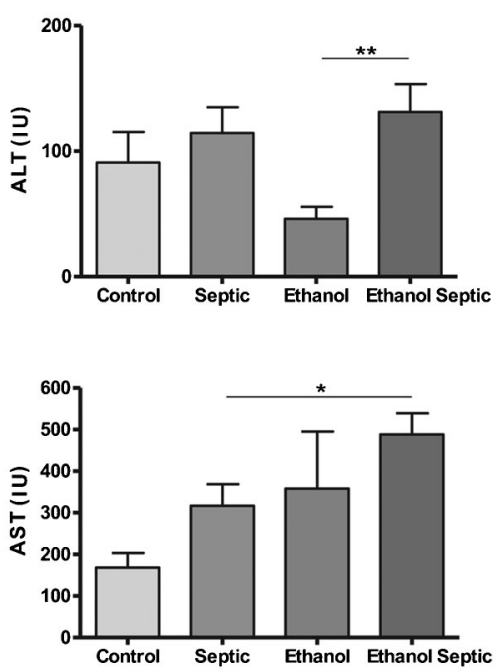

Figure 3. Alanine aminotransferase (ALT) and aspartate aminotransferase (AST) levels were increased in animals exposed to ethanol and subjected to sepsis. Control $(n=12)$ and septic $(n=20)$ groups received water, and ethanol $(n=12)$ and ethanol septic $(n=25)$ groups received $10 \%$ ethanol solution for 4 weeks. Septic and ethanol septic groups underwent septic procedure while control and ethanol groups received normal saline injected intraperitoneally. Whole blood was obtained by cardiac puncture $8 \mathrm{~h}$ after sepsis induction or saline injection in animals in estrus phase. Data are reported as means \pm SE for at least 12 animals. ALT: ${ }^{* *} \mathrm{P}=0.007$. AST: ${ }^{*} \mathrm{P}=0.03$ (Mann-Whitney test). 

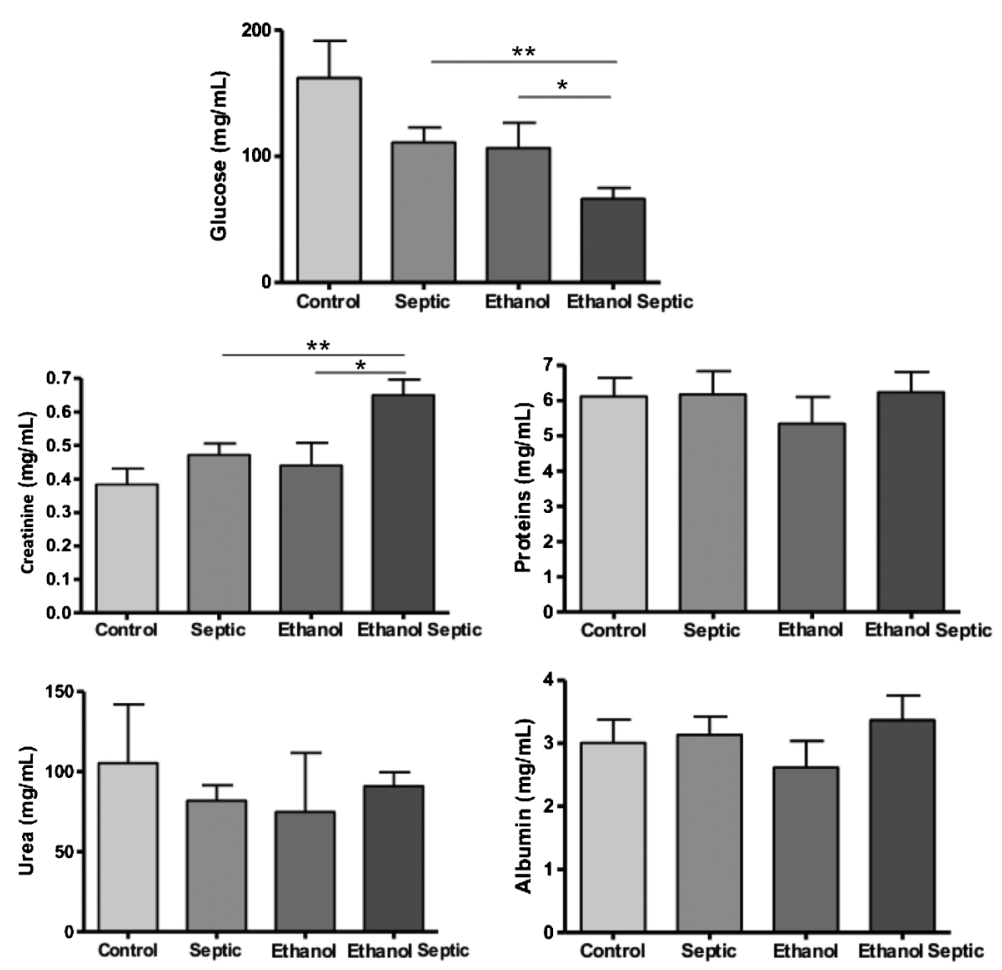

Figure 4. Levels of glucose and creatinine were altered in animals exposed to ethanol and subjected to sepsis. Control $(n=12)$ and septic $(n=20)$ groups received water, and ethanol $(n=12)$, and ethanol septic $(n=25)$ groups received $10 \%$ ethanol solution for 4 weeks. Septic and ethanol septic groups underwent septic procedure while control and ethanol groups received normal saline injected intraperitoneally. Whole blood was obtained by cardiac puncture $8 \mathrm{~h}$ after sepsis induction or saline injection in animals in estrus phase. Data are reported as means $\pm \mathrm{SE}$ for at least 12 animals. Glucose: ${ }^{* *} \mathrm{P}=0.008 ;{ }^{*} \mathrm{P}=0.04$. Creatinine: ${ }^{* *} \mathrm{P}=0.01$; ${ }^{*} \mathrm{P}=0.03$ (Mann-Whitney test). There were no significant differences in proteins, urea and albumin levels between groups. source of liquid for 4 weeks (13). Mortality was directly correlated to ethanol consumption, that is, rats that voluntarily consumed more ethanol $(>11 \mathrm{~g} / \mathrm{kg})$ died earlier. In the present study, mean consumption of ethanol was $10.9 \mathrm{~g} / \mathrm{kg}$, and yet the survival rate differed from that found in males. Taken together, these data suggest a possible protective role of the female hormone profile in the sepsis process.

Acute ethanol intake plus trauma can decrease TNF- $\alpha$ production during the very early postinjury (0-3 days) period. However, TNF- $\alpha$ production by ethanol-exposed patients became hyperelevated late postinjury ( $>9$ days). Elevated TNF- $\alpha$ levels such as these may contribute to post-trauma immunosuppression after acute ethanol use (14). Although pro-inflammatory cytokines are considered to be dangerous, causing organ failure and apoptosis of immune system cells (15), they also have essential beneficial effects. Blocking TNF- $\alpha$ worsens survival from peritonitis in animal models and clinical studies $(16,17)$. The role of TNF- $\alpha$ in combating infection has been underscored by the finding that sepsis and other infectious complications have been observed in patients with rheumatoid arthritis treated with $\mathrm{TNF}-\alpha$ antagonists (18). Furthermore, repetitive ip injection of sublethal doses of recombinant human TNF- $\alpha$ in rats undergoing cecal ligation and puncture (CLP) 1 day after the treatment period resulted in a significant reduction in mortality compared with control rats previously unexposed to TNF- $\alpha$ (19). In this study, the TNF- $\alpha$ increase in the ethanol septic group compared with the septic group confirms the pro-inflammatory beneficial role of TNF- $\alpha$ in the acute phase of sepsis.

Multiple studies have demonstrated that elevated serum IL-6 correlates with increased mortality $(20,21)$. In the CLP model of sepsis, animals with the highest mortality have the highest levels of IL-6 (22). In rodent models, the association between increased IL-6 levels after burn, sepsis or trauma-hemorrhage and cardiac dysfunction combined with poor outcome has also been identified (23). Our results demonstrated that animals that had ethanol consumption and sepsis had lower IL-6 and MIF levels. These results can be linked to their improved survival, especially because MIF, similar to IL-6, also contributes to the pathogenesis of sepsis (24). Blockade of MIF with specific neutralizing monoclonal antibodies protected mice from lethal sepsis induced by CLP (survival was $81 \%$ in antibody-treated mice and $31 \%$ in control mice). This treatment was successful even when antibodies were administered $4.5 \mathrm{~h}$ after CLP (survival was $61 \%$ in antibody-treated mice and $5 \%$ in control mice) (25).

In male rats treated using the same experimental model, sepsis significantly increased levels of the proinflammatory cytokines IL-6 and TNF- $\alpha$. No significant differences were found in IL-4 and IL-13. Ethanol significantly decreased the effect of sepsis in the levels of IL-6 and TNF- $\alpha$ (13).

Biochemical parameters demonstrated that rats in the ethanol septic group had lower glucose levels than those 
in the septic and ethanol groups. Ethanol can cause hypoglycemia by inhibiting gluconeogenesis, reducing glucose synthesis, and impairing its transport to the liver perivascular space (26). Hypoglycemia in sepsis may have occurred as a result of lethargy of the animals, leading to lower water and food consumption, and may have been one of the causes of the decrease in prolonged survival in ethanol septic rats at $48 \mathrm{~h}$ compared with $24 \mathrm{~h}$.

Mortality from sepsis is increased when complicated by acute kidney injury (27). However, the association between ethanol consumption and renal function is poorly understood. There is evidence that chronic ethanol consumption may cause direct damage to the kidneys (28). In this study, increased creatinine levels in sepsis shows that there was a decrease in glomerular filtration, probably caused by ethanol. Similarly, liver injury can increase the risk of septic shock, which is very common in alcoholics (29). The higher levels of ALT in the ethanol sepsis group compared to the ethanol group and the increased levels of AST in the ethanol sepsis group compared to the sepsis group are in accordance with other studies. However, the liver injury indicated by the AST and ALT levels was probably not enough to increase mortality in this group of animals.

Regarding the female hormones, chronic exposure to ethanol alters the production of estrogen and hence ethanol's effects on immunity could involve an indirect mechanism in which ethanol alters hormone levels and, in turn, the hormones regulate immune responses. There is evidence of a protective role of estrogen in trauma and sepsis. In male rodents, administration of $17 \beta$-estradiol (estrogen) after trauma-hemorrhage normalized the

\section{References}

1. Yu HP, Chaudry IH. The role of estrogen and receptor agonists in maintaining organ function after trauma-hemorrhage. Shock 2009; 31: 227-237, doi: 10.1097/SHK. 0b013e31818347e7.

2. Goral J, Karavitis J, Kovacs EJ. Exposure-dependent effects of ethanol on the innate immune system. Alcohol 2008; 42: 237-247, doi: 10.1016/j.alcohol.2008.02.003.

3. O'Brien JM Jr, Lu B, Ali NA, Martin GS, Aberegg SK, Marsh $\mathrm{CB}$, et al. Alcohol dependence is independently associated with sepsis, septic shock, and hospital mortality among adult intensive care unit patients. Crit Care Med 2007; 35: 345-350, doi: 10.1097/01.CCM.0000254340.91644.B2.

4. Abraham E, Matthay MA, Dinarello CA, Vincent JL, Cohen $\mathrm{J}$, Opal SM, et al. Consensus conference definitions for sepsis, septic shock, acute lung injury, and acute respiratory distress syndrome: time for a reevaluation. Crit Care Med 2000; 28: 232-235, doi: 10.1097/00003246-20000100000039 .

5. Hoyert DL, Arias E, Smith BL, Murphy SL, Kochanek KD. Deaths: final data for 1999. Natl Vital Stat Rep 2001; 49: 1113.

6. Frank J, Witte K, Schrodl W, Schutt C. Chronic alcoholism enhanced pro-inflammatory cytokine release from several cell types such as Kupffer cells and cardiomyocytes. Estrogen treatment after trauma-hemorrhage was also able to restore cardiac function, presumably because of the normalization of IL-6 release (23). The increase in TNF- $\alpha$ production, detected in our study, could explain the decreased CFU numbers observed in experiment 3 in the ethanol septic group, and is probably an additional factor increasing survival rates in this group of animals.

Macrophages are the first line of defense against bacterial colonization of ascites fluid (30). The increase in TNF- $\alpha$ production detected in our study may be related to the activation of macrophages and consequent early bacterial clearance. This rationale could explain the observed mortality delay in the ethanol septic group. However, this mechanism was not able to prevent the effects of other cytokines on multiple organ failure.

We, therefore, conclude that delayed mortality associated with ethanol consumption in the ethanol sepsis group could be attributed to female characteristics, most probably sex hormones. However, we cannot clarify whether the cytokine modulation observed in this study, which also exerted a protective role in sepsis, was due directly to ethanol consumption or to increased levels of sex hormones. Further investigation with estrogen replacement in ovariectomized female animals exposed to ethanol could be useful to evaluate this issue.

\section{Acknowledgments}

We are grateful to Mr. R.A. Laurindo and Mr. E.F. Assis for their technical assistance.

causes deleterious conditioning of innate immunity. Alcohol Alcohol 2004; 39: 386-392, doi: 10.1093/alcalc/agh083.

7. Choudhry MA, Schwacha MG, Hubbard WJ, Kerby JD, Rue $\mathrm{LW}$, Bland $\mathrm{KI}$, et al. Gender differences in acute response to trauma-hemorrhage. Shock 2005; 24 (Suppl 1): 101-106, doi: 10.1097/01.shk.0000191341.31530.5e.

8. Angele MK, Schwacha MG, Ayala A, Chaudry IH. Effect of gender and sex hormones on immune responses following shock. Shock 2000; 14: 81-90, doi: 10.1097/00024382200014020-00001.

9. George RL, McGwin G Jr, Windham ST, Melton SM, Metzger J, Chaudry IH, et al. Age-related gender differential in outcome after blunt or penetrating trauma. Shock 2003; 19: 28-32, doi: 10.1097/00024382-200301000-00006.

10. Kher A, Wang M, Tsai BM, Pitcher JM, Greenbaum ES, Nagy RD, et al. Sex differences in the myocardial inflammatory response to acute injury. Shock 2005; 23: 110, doi: 10.1097/01.shk.0000148055.12387.15

11. Olsen NJ, Kovacs WJ. Gonadal steroids and immunity. Endocr Rev 1996; 17: 369-384.

12. Yokoyama Y, Schwacha MG, Bland KI, Chaudry IH. Effect of estradiol administration on splanchnic perfusion after 
trauma-hemorrhage and sepsis. Curr Opin Crit Care 2003; 9: 137-142, doi: 10.1097/00075198-200304000-00010.

13. Barros FR, Castro-Faria-Neto HC, Castro CL, Aguiar Nemer AS, Rocha EM, Silva-Fonseca VA. Effects of chronic ethanol consumption in experimental sepsis. Alcohol Alcohol 2012; 47: 677-682, doi: 10.1093/alcalc/ags081.

14. Szabo G, Mandrekar P, Verma B, Isaac A, Catalano D. Acute ethanol consumption synergizes with trauma to increase monocyte tumor necrosis factor alpha production late postinjury. J Clin Immunol 1994; 14: 340-352, doi: 10.1007/BF01546318.

15. Hotchkiss RS, Tinsley KW, Swanson PE, Schmieg RE Jr, Hui JJ, Chang KC, et al. Sepsis-induced apoptosis causes progressive profound depletion of B and CD4 + T lymphocytes in humans. J Immunol 2001; 166: 6952-6963.

16. Echtenacher B, Weigl K, Lehn N, Mannel DN. Tumor necrosis factor-dependent adhesions as a major protective mechanism early in septic peritonitis in mice. Infect Immun 2001; 69: 3550-3555, doi: 10.1128/IAI.69.6.3550-3555.2001.

17. Reinhart K, Karzai W. Anti-tumor necrosis factor therapy in sepsis: update on clinical trials and lessons learned. Crit Care Med 2001; 29: S121-S125, doi: 10.1097/00003246200107001-00037.

18. Keane J, Gershon S, Wise RP, Mirabile-Levens E, Kasznica $\mathrm{J}$, Schwieterman WD, et al. Tuberculosis associated with infliximab, a tumor necrosis factor alpha-neutralizing agent. $N$ Engl J Med 2001; 345: 1098-1104, doi: 10.1056/ NEJMoa011110.

19. Alexander HR, Sheppard BC, Jensen JC, Langstein HN, Buresh CM, Venzon D, et al. Treatment with recombinant human tumor necrosis factor-alpha protects rats against the lethality, hypotension, and hypothermia of Gram-negative sepsis. J Clin Invest 1991; 88: 34-39, doi: 10.1172/JCI115298.

20. Vianna RC, Gomes RN, Bozza FA, Amancio RT, Bozza PT, David CM, et al. Antibiotic treatment in a murine model of sepsis: impact on cytokines and endotoxin release. Shock 2004; 21: 115-120, doi: 10.1097/01.shk.0000111828.07309. 26.

21. Remick DG, Bolgos GR, Siddiqui J, Shin J, Nemzek JA. Six at six: interleukin- 6 measured $6 \mathrm{~h}$ after the initiation of sepsis predicts mortality over 3 days. Shock 2002; 17: 463467, doi: 10.1097/00024382-200206000-00004.

22. Ebong SJ, Call DR, Bolgos G, Newcomb DE, Granger JI, O'Reilly M, et al. Immunopathologic responses to non-lethal sepsis. Shock 1999; 12: 118-126, doi: 10.1097/00024382199908000-00005.

23. Yang S, Zheng R, Hu S, Ma Y, Choudhry MA, Messina JL, et al. Mechanism of cardiac depression after traumahemorrhage: increased cardiomyocyte IL-6 and effect of sex steroids on IL-6 regulation and cardiac function. Am J Physiol Heart Circ Physiol 2004; 287: H2183-H2191, doi: 10.1152/ajpheart.00624.2003.

24. Calandra T, Roger T. Macrophage migration inhibitory factor: a regulator of innate immunity. Nat Rev Immunol 2003; 3: 791-800, doi: 10.1038/nri1200

25. Calandra T, Echtenacher B, Roy DL, Pugin J, Metz CN, Hultner L, et al. Protection from septic shock by neutralization of macrophage migration inhibitory factor. Nat Med 2000; 6: 164-170, doi: 10.1038/72262.

26. Van Horn CG, Cunningham CC. Contributions of dietary carbohydrate and ethanol to alterations in liver glycogen levels and glycolytic activity. Alcohol 1999; 19: 139-144, doi: 10.1016/S0741-8329(99)00030-0.

27. Uchino S, Bellomo R, Morimatsu H, Morgera S, Schetz M, Tan I, et al. External validation of severity scoring systems for acute renal failure using a multinational database. Crit Care Med 2005; 33: 1961-1967, doi: 10.1097/ 01.CCM.0000172279.66229.07.

28. Vamvakas S, Teschner M, Bahner U, Heidland A. Alcohol abuse: potential role in electrolyte disturbances and kidney diseases. Clin Nephrol 1998; 49: 205-213.

29. Leibovici L, Drucker M, Konigsberger H, Samra Z, Harrari S, Ashkenazi S, et al. Septic shock in bacteremic patients: risk factors, features and prognosis. Scand J Infect Dis 1997; 29: 71-75, doi: 10.3109/00365549709008668.

30. Dunn DL, Barke RA, Knight NB, Humphrey EW, Simmons $\mathrm{RL}$. Role of resident macrophages, peripheral neutrophils, and translymphatic absorption in bacterial clearance from the peritoneal cavity. Infect Immun 1985; 49: 257264. 